\title{
SUPER-RESOLUÇÃO REGULARIZADA E SIMULTÂNEA DE SEQÜÊNCIA DE IMAGENS
}

\author{
Marcelo Victor Wüst Zibetti e Joceli Mayer
}

Resumo - Este trabalho apresenta um nova classe de algoritmos de super-resolução de imagens. Nesta classe de algoritmos todos os quadros de uma seqüência de imagens são estimados simultaneamente através da minimização iterativa de uma função de custo regularizada. Similarmente a outras técnicas de super-resolução, a abordagem proposta explora a correlação existente entre os quadros com o movimento compensado. Desta forma, consegue-se recuperar detalhes perdidos no processo de aquisição e obter uma seqüência com maior resolução. A técnica proposta obteve um melhor desempenho, em relação às outras técnicas, utilizando as equações que expressam o movimento somente no termo $a$ priori da função de custo. Esta abordagem original permite a produção de uma seqüência de imagens com maior fidelidade e, ao mesmo tempo, com menor custo computacional quando comparada com os demais algoritmos existentes na literatura. $\mathrm{O}$ isolamento no termo a priori das expressões que descrevem a correlação das imagens na trajetória de movimento permite que os efeitos dos erros de movimento sejam melhor controlados e, conseqüentemente, resulta em uma maior robustez a estes erros. O desempenho da abordagem proposta foi contrastada com o desempenho dos algoritmos encontrados na literatura. Nos experimentos comparativos utilizou-se a norma Euclidiana, a qual apresenta baixo custo computacional, e também a norma Huber, a qual proporciona imagens com bordas mais nítidas e com uma robustez superior aos erros de movimento.

Palavras-chave: Processamento de imagens, superresolução de vídeo, restauração regularizada de imagens.

\begin{abstract}
This work presents a new class of algorithms for super-resolution of images. In this new class of algorithms all frames of a sequence of images are simultaneously estimated by employing an iterative minimization of a regularized cost function. Similarly to other super-resolution techniques, the proposed approach exploits the existent correlation among the motion compensated frames. This correlated information helps to recover the details lost in the acquisition process and to obtain a image sequence with improved resolution. The proposed method achieved a better performance, when compared to other methods, by using the motion equations only in the prior term of the cost function. This original approach allows the production of an image sequence with higher fidelity and, at the same time, with lower computational complexity when compared with other algorithms in the literature. The isolation of the expressions that describes the correlation in the motion trajectory allows an increased
\end{abstract}

Este trabalho tem o apoio do CNPQ, no. 140543/2003-1

Marcelo Victor Wüst Zibetti e Joceli Mayer estão com a Universidade Federal de Santa Catarina (E-mails: marcelo.zibetti@terra.com.br, mayer@eel.ufsc.br) control over the motion errors, and as a consequence, it was achieved an increased robustness. The performance of the proposed method were compared with other methods in the literature. In the comparative experiments it was considered the Euclidean norm, which is used to achieve low computational complexity, and the Huber norm, which is used to produce image with sharp edges and higher robustness to errors that occurs in the motion.

Keywords: Image processing, super-resolution for video sequences, regularized image restoration.

\section{INTRODUÇÃO}

Na maioria das aplicações de Processamento de Imagens é desejável que o sistema de aquisição apresente a maior resolução possível e introduza uma quantidade mínima de distorções devido às imperfeições óticas e aos ruídos inerentes ao sistema. Entretanto, a densidade dos sensores empregados em sistemas de aquisição como câmeras, filmadoras e escaneadores digitais é limitada e, assim como a qualidade do sistema ótico implementado, estão diretamente associadas ao custo do sistema. Uma alternativa para aumentar a resolução e a qualidade das imagens adquiridas mantendo o custo do sistema de aquisição, é a utilização de técnicas de processamento digital das imagens adquiridas visando ao aumento de resolução.

Estas técnicas, chamadas de super-resolução (SR), proporcionam imagens com resolução superior à fornecida pelo dispositivo de aquisição [1, 2, 3, 4, 5]. Em aplicações que envolvem seqüências de imagens, como vídeo, as técnicas de SR operam em todos os quadros visando criar uma seqüência de imagens de alta resolução (HR - High-Resolution). Existem diversas aplicações para técnicas de SR, incluindo a produção de vídeo de alta definição, a recuperação de vídeos capturados por dispositivos antigos e a remoção de distorções causadas pela utilização de lentes e sensores de baixa qualidade.

As técnicas de SR procuram remover possíveis distorções geradas no processo de aquisição, realçando, por exemplo, as bordas e os detalhes dos objetos. O objetivo destas técnicas é produzir imagens com a melhor qualidade possível considerando as limitações de um determinado sistema de aquisição. As técnicas SR alcançam estas melhorias considerando a informação correlacionada existente na sequiência de imagens capturadas e utilizando modelos que descrevem o comportamento espacial das imagens da seqüência.

A maioria das técnicas na literatura $[1,2,4,5]$ utilizam um conjunto de imagens capturadas para recuperar os detalhes das imagens HR. Assume-se que as imagens capturadas, 
denominadas de imagens de baixa resolução (LR - LowResolution), têm grande similaridade entre si. Porém, uma pequena diferença de informação existente em cada imagem capturada proporciona uma nova informação para a recuperação dos detalhes na imagem HR. A fonte mais comum desta diferença de informação é o movimento de "subpixel" $[1,4,2]$, o qual ocorre quando o movimento existente entre as imagens é fracionário com respeito a grade de baixa resolução, conforme ilustrado na Figura 1. Para que esta informação seja explorada adequadamente é necessário que se conheça o movimento existente com precisão.

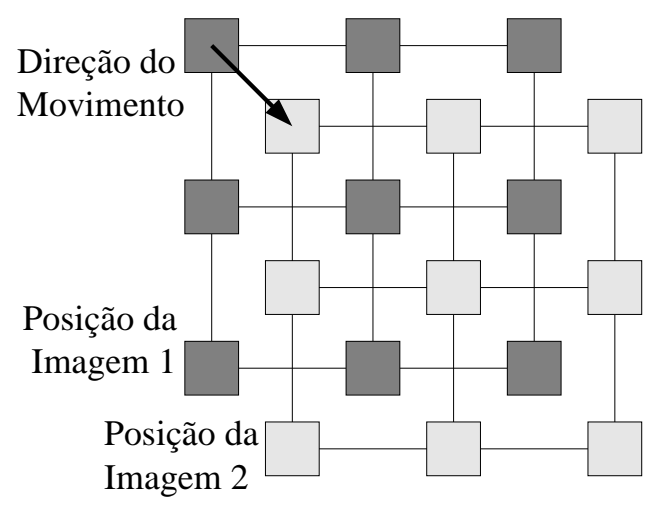

Figura 1. Ilustração do movimento de subpixel.

Entretanto, em regiões da seqüência onde existem movimentos complexos, as limitações dos modelos de movimento adotados, que podem reproduzir apenas movimentos de translação e rotação, por exemplo, e as limitações do algoritmo de estimação de movimento empregado produzem uma representação inadequada do movimento real existente na seqüência de imagens. Nestas situações é inevitável a ocorrência de erros na representação do movimento. Portanto, para que se possa aproveitar a informação do movimento de subpixel nas regiões onde ele é adequadamente representado, é necessário que o algoritmo de super-resolução seja robusto aos erros existentes nas regiões onde ele não é adequadamente representado. Uma vez que o movimento seja corretamente estimado e os erros sejam apropriadamente tratados, pode-se estimar a seqüência de imagens HR, utilizando o conjunto de imagens LR capturadas em um processo de super-resolução.

Os algoritmos de super-resolução podem ser classificados em três classes, de acordo com a forma de execução da superresolução:

- Métodos Tradicionais: são métodos que aplicam as técnicas de SR visando a obtenção de uma única imagem da seqüência HR. O procedimento pode ser aplicado repetidamente, visando a obtenção das demais imagens HR da seqüência de forma independente [1, 2, 4, 6, 7].

- Métodos Seqüenciais: são métodos que visam a super-resolução de uma imagem HR da seqüência por processo. Contudo, se diferenciam dos métodos tradicionais, pois aproveitam as imagens HR previamente estimadas para realizar a estimativa das imagens HR subseqüientes $[8,9]$.
- Métodos Simultâneos: são métodos que estimam toda a sequiência de imagens em um único processo, onde todas as imagens HR são estimadas simultaneamente [10, 11].

Os métodos tradicionais de super-resolução foram os primeiros a serem aplicados em seqüências de imagens. Porém, estes métodos estimam uma imagem da seqüência de cada vez sem aproveitar as imagens HR previamente estimadas. O algoritmo seqüencial de [8] foi introduzido com o objetivo de reduzir o custo computacional total aproveitando as imagens previamente estimadas, porém a qualidade das estimativas foi comprometida, sendo inferior a obtida pelos métodos tradicionais.

Por outro lado, os métodos simultâneos foram desenvolvidos para buscar uma qualidade superior nos resultados. Isso é obtido através da melhor utilização da informação sobre o movimento presente na seqüência de imagens. Todavia, o custo computacional para a obtenção desta melhoria no primeiro algoritmo simultâneo proposto [10] era muito maior do que o dos métodos tradicionais.

Neste trabalho é apresentada uma nova classe de algoritmos simultâneos de super-resolução. São explicitadas as características dos métodos simultâneos que proporcionam a superioridade em relação aos métodos tradicionais. Isso permitiu o desenvolvimento de uma nova classe de algoritmos simultâneos, proposto neste trabalho, que necessitam um número reduzido de termos na função de custo empregada para solucionar o problema. Os algoritmos propostos mantém a qualidade dos métodos simultâneos anteriores [10], mas com um custo computacional menor. Em alguns casos, o custo computacional chega a ser menor do que o custo dos métodos tradicionais. Além disso, as equações que envolvem o modelo de movimento são isoladas do modelo de aquisição neste novo algoritmo, permitindo um tratamento diferenciado para as regiões onde ocorrem grandes erros de movimento. Assim, o método proposto torna-se mais robusto aos erros de estimativa de movimento.

Na Seção 2 são apresentados os modelos utilizados em super-resolução. Na Seção 3 tem-se uma revisão de importantes algoritmos de super-resolução baseados nos métodos regularizados $[1,2]$, a classe na qual o método proposto é baseado. A nova classe de algoritmos simultâneos é apresentada na Seção 4, juntamente com o método de otimização sugerido. Na Seção 5 é apresentada uma análise onde os algoritmos são contrastados quanto à qualidade obtida, quanto ao custo computacional e quanto à robustez a grandes erros de movimento. Na Seção 6 são apresentadas simulações com seqüências de imagens reais e na Seção 7 são oferecidas as conclusões.

\section{MODELOS USADOS EM SUPER- RESOLUÇÃO}

Nesta seção são apresentados os modelos utilizados nos algoritmos de super-resolução. Estes modelos descrevem os processos associados às degradações, ao movimento relativo e à geração das imagens. Os modelos considerados são: o modelo de imagem, o modelo de aquisição de imagem, o modelo de movimento entre as imagens e o modelo combi- 
nado de aquisição e movimento, o qual é utilizado em alguns algoritmos.

\subsection{AQUISIÇÃO DE IMAGEM}

O modelo de aquisição descreve as transformações que ocorrem com as imagens HR durante o processo de aquisição. Neste modelo, os dados observados são as imagens LR. O modelo é descrito pela seguinte equação:

$$
\mathbf{g}_{k}=\mathbf{D}_{k} \mathbf{f}_{k}+\boldsymbol{\eta}_{k}
$$

onde $\mathrm{g}_{k}$ é um vetor, de tamanho $N \times 1$, que representa o quadro LR adquirido no instante $k$. Os elementos de $\mathbf{g}_{k}$ correspondem aos pixels da imagem LR, $g_{k}\left[n_{1}, n_{2}\right]$, ordenados lexicograficamente. Onde $N=N_{1} N_{2}$ é o número de pixels na imagem LR, sendo $N_{1} \times N_{2}$ o tamanho da imagem LR na direção horizontal e vertical, respectivamente. $\mathrm{O}$ vetor $\mathbf{f}_{k}$, de tamanho $M \times 1$, representa a imagem HR, $f_{k}\left[m_{1}, m_{2}\right]$, de tamanho $M_{1} \times M_{2}$, com $M=M_{1} M_{2}$ pixels ordenados lexicograficamente, onde $N \leq M$. O vetor $\boldsymbol{\eta}_{k}$ representa o ruído de aquisição, o qual é normalmente modelado como um ruído Gaussiano branco, de variância $\sigma_{\eta}^{2}$ para todos os quadros.

A matriz $\mathbf{D}_{k}$, de tamanho $N \times M$, representa o sistema de aquisição para a imagem $f_{k}$. Cada linha de $\mathbf{D}_{k}$ representa a Função de Aquisição do respectivo pixel na imagem LR, e contém a ponderação de cada pixel da imagem HR na formação do pixel da imagem LR. A matriz $\mathbf{D}_{k}$ é uma versão discretizada do sistema de aquisição contínuo [12]. O processo de aquisição degrada a imagem, e $\mathbf{D}_{k}$ representa as degradações sofridas na aquisição. As degradações são divididas em distorção ótica, gerada nas lentes e fotossensores, e a distorção devido à subamostragem, a qual depende do arranjo geométrico dos sensores. A subamostragem implica na redução do número de amostras ou pixels. Este modelo é denominado temporalmente coincidente, porque a imagem HR e a imagem LR capturada correspondem ao mesmo instante temporal.

\subsection{MOVIMENTO ENTRE IMAGENS}

O modelo de movimento descreve a transformação causada pelo movimento entre as imagens da sequiência. Assume-se que a imagem no instante temporal $k$ pode ser composta pela imagem do instante temporal $j$, com o movimento compensado, mais uma nova informação, que não pode ser obtida da imagem no instante $j$. A equação que descreve o modelo é:

$$
\mathbf{f}_{k}=\mathbf{M}_{k, j} \mathbf{f}_{j}+\mathbf{e}_{k, j}
$$

onde $\mathbf{f}_{k}$ e $\mathbf{f}_{j}$ são vetores que representam as imagens $f_{k}\left[m_{1}, m_{2}\right]$ e $f_{j}\left[m_{1}, m_{2}\right]$ nos instantes temporais $k$ e $j$, respectivamente. A matriz $\mathbf{M}_{k, j}$, de tamanho $M \times M$, representa a transformação de movimento. Esta matriz é, normalmente, bastante esparsa [13]. Uma linha $m$, qualquer, é quase toda composta por zeros, exceto por: 1) um elemento com valor unitário, na coluna $n$, que representa o deslocamento do pixel $n$ em $\mathbf{f}_{j}$ para posição $m$ em $\mathbf{f}_{k} ; 2$ ) poucos elementos não-nulos que correspondem a combinação de determinados pixels (interpolação) de $\mathbf{f}_{j}$ para formar o pixel $m$ em $\mathbf{f}_{k}$. Isso ocorre em movimentos cujo deslocamento do pixel é fracionário na grade de alta resolução.

A matriz $\mathbf{M}_{k, j}$ pode ser uma versão discretizada do operador contínuo de transformação de movimento, no caso de movimento paramétrico, ou pode ser criada a partir de um campo discreto de vetores de movimento $[14,15]$. O vetor $\mathbf{e}_{k, j}$ é chamado de erro de movimento e representa a nova informação que não pode ser obtida da imagem no instante $j$.

Esta nova informação pode ser dividida em duas partes:

1. Pequenas diferenças na imagem com o movimento compensado, as quais podem ser originadas por pequenos erros na estimação dos parâmetros de movimento, limitações do operador discretizado ou do campo discreto de vetores em representar o movimento contínuo, ou ainda, outras variações temporais não relacionadas ao movimento.

2. Erros de grande magnitude, considerados como outliers $^{1}$ [17]. No contexto do movimento, um outlier é geralmente uma região ou um objeto que recebeu oclusão pelo movimento de outros objetos, objetos que aparecem repentinamente na cena ou regiões que sofrem um movimento não esperado. O movimento translacional também gera outliers nas bordas das imagens.

Os outliers introduzem distorções nas imagens estimadas. É necessário utilizar algoritmos robustos [16] aos outliers, se possível, ou é preciso detectá-los, usando técnicas como em $[17,15]$, e eliminá-los. Assumindo que os outliers foram corretamente detectados, a seguinte equação é utilizada, onde os outliers são removidos:

$$
\begin{aligned}
& \mathbf{I}_{k, j} \mathbf{f}_{k}=\mathbf{I}_{k, j}\left(\mathbf{M}_{k, j} \mathbf{f}_{j}+\mathbf{e}_{k, j}\right) \\
& \mathbf{I}_{k, j} \mathbf{f}_{k}=\mathbf{I}_{k, j} \mathbf{M}_{k, j} \mathbf{f}_{j}+\mathbf{I}_{k, j} \mathbf{e}_{k, j} \\
& \overline{\mathbf{f}}_{k, j}=\overline{\mathbf{M}}_{k, j} \mathbf{f}_{j}+\overline{\mathbf{e}}_{k, j}
\end{aligned}
$$

onde a matriz $\mathbf{I}_{k, j}$, de tamanho $Q \times M$, sendo $Q \leq M$, possui apenas um elemento unitário em cada linha, exatamente nas colunas correspondentes aos elementos assumidos como inliers, i.e., pixels observados em ambas imagens através do movimento. Esta matriz preserva os inliers e remove os outliers do sistema. $\mathbf{I}_{k, j}$ é gerado a partir do processo de detecção dos outliers. Os vetores $\overline{\mathbf{f}}_{k, j}$ e $\overline{\mathbf{e}}_{k, j}$, de tamanho $Q \times 1$, são compostos apenas pelos $Q$ elementos considerados inliers de $\mathbf{f}_{k}$ e $\mathbf{e}_{k, j}$, respectivamente. A matriz $\overline{\mathbf{M}}_{k, j}$ é uma matriz de dimensão $Q \times M$, similar a $\mathbf{M}_{k, j}$, onde as linhas correspondentes aos outliers são removidas.

Na Figura 2 são apresentadas duas imagens, $\mathbf{f}_{k}$ e $\mathbf{f}_{j}$, a nova informação, $\mathbf{e}_{k, j}=\mathbf{f}_{k}-\mathbf{M}_{k, j} \mathbf{f}_{j}$, e os pixels considerados inliers. Pode-se identificar facilmente os grandes erros, ou outliers, nos cantos da imagem $\mathbf{e}_{k, j}$, e os pequenos erros devido ao movimento de pixels, ou inliers, na região central da imagem $\mathbf{e}_{k, j}$. Na imagem formada após a detecção de inliers e outliers pode-se observar a região de inliers em branco, a qual é preservada, e a região de outliers em preto, a qual é removida.

\footnotetext{
${ }^{1} \mathrm{O}$ termo outlier foi, inicialmente, utilizado em Estatística. Na literatura da área de Estatística um outlier é um elemento ou uma medida ruim, geralmente um erro de grande magnitude, que não segue o modelo assumido [16].
} 


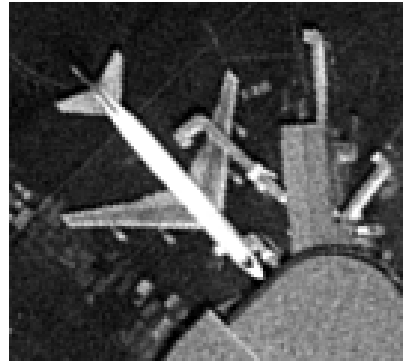

(a) Imagem $\mathbf{f}_{k}$

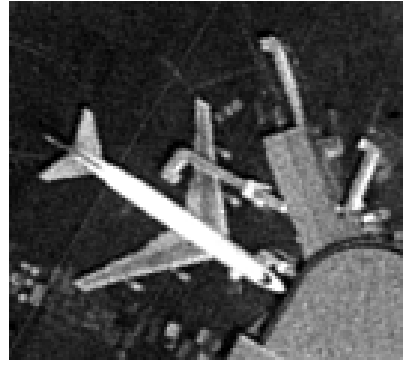

(b) Imagem $\mathbf{f}_{j}$

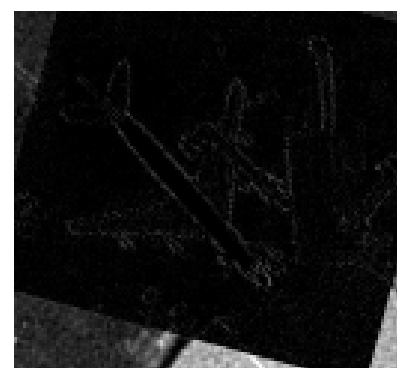

(c) Imagem $\mathbf{e}_{k, j}$

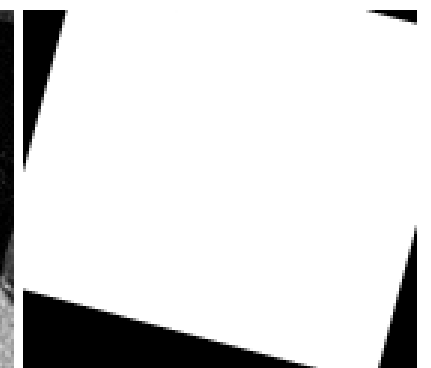

(d) Mapeamento dos pixels considerados inliers (em branco)
Figura 2. Ilustração da nova informação temporal $\mathbf{e}_{k, j}$.

\subsection{MODELO DE AQUISIÇÃO E DE MOVI- MENTO COMBINADOS}

Os algoritmos de super-resolução que seguem a forma tradicional [2], alguns dos algoritmos seqüenciais [9] e o algoritmo simultâneo proposto em [10] utilizam o modelo de aquisição combinado com o modelo de movimento. O modelo combinado é utilizado para relacionar uma imagem LR à uma outra imagem HR, em um instante temporal diferente. Esse modelo é descrito pela equação:

$$
\begin{aligned}
& \mathbf{g}_{k}=\mathbf{D}_{k}\left(\mathbf{M}_{k, j} \mathbf{f}_{j}+\mathbf{e}_{k, j}\right)+\boldsymbol{\eta}_{k} \\
& \mathbf{g}_{k}=\mathbf{D}_{k} \mathbf{M}_{k, j} \mathbf{f}_{j}+\mathbf{D}_{k} \mathbf{e}_{k, j}+\boldsymbol{\eta}_{k} \\
& \mathbf{g}_{k}=\mathbf{D}_{k} \mathbf{M}_{k, j} \mathbf{f}_{j}+\boldsymbol{\epsilon}_{k, j}+\boldsymbol{\eta}_{k} \\
& \mathbf{g}_{k}=\mathbf{C}_{k, j} \mathbf{f}_{j}+\boldsymbol{\varepsilon}_{k, j}
\end{aligned}
$$

onde $\mathbf{C}_{k, j}=\mathbf{D}_{k} \mathbf{M}_{k, j}$ é a matriz de transformação conjunta de movimento e aquisição. O erro $\varepsilon_{k, j}$ do modelo combinado é uma soma de dois erros de naturezas diferentes: o ruído de aquisição $\boldsymbol{\eta}_{k}$ e o erro de movimento projetado nos dados $\boldsymbol{\epsilon}_{k, j}$, o qual pode conter outliers.

Por causa da existência dos outliers, ou é necessário usar um algoritmo robusto ou é necessário um procedimento de detecção e eliminação de outliers. Caso os outliers sejam detectados e removidos, como em (3), a seguinte equação para o modelo combinado é adotada:

$$
\overline{\mathbf{g}}_{k, j}=\overline{\mathbf{C}}_{k, j} \mathbf{f}_{j}+\bar{\varepsilon}_{k, j}
$$

onde os vetores $\overline{\mathbf{g}}_{k, j}$ e $\bar{\varepsilon}_{k, j}$ são vetores que podem ter menos elementos que os vetores $\mathbf{g}_{k}$ e $\varepsilon_{k, j}$, onde os elementos correspondentes aos pixels da imagem LR considerados outliers são removidos. A matriz $\overline{\mathbf{C}}_{k, j}$ é uma matriz equivalente a matriz $\mathbf{C}_{k, j}$, porém com as linhas referentes aos outliers removidas. Este procedimento é similar ao descrito em (3) e utilizado em [10, 15, 18].
Este modelo combinado é geralmente chamado de temporalmente não-coincidente, porque a imagem LR é associada ao instante temporal $k$ enquanto que a imagem HR é associada ao instante temporal $j$.

\subsection{MODELOS DE IMAGEM}

Em geral, super-resolução é um problema mal-posto ${ }^{2}$, seja porque tem infinitas soluções possíveis ou porque a solução possui grande sensibilidade ao ruído [2, 4, 19]. Para resolver este problema e obter uma solução única e estável, os algoritmos de super-resolução usam informações adicionais sobre a imagem. Nos algoritmos regularizados de superresolução derivados de estimação estatística Bayesiana, esta informação adicional é definida na forma de uma distribuição a priori [21, 22]. Nos algoritmos regularizados derivados de métodos determinísticos esta informação é tratada geralmente como uma penalidade de regularização [12, 19, 23].

A informação adicional mais comumente assumida é de que as imagens são suaves. Em outras palavras, as variações na intensidade de $\mathbf{f}_{k}$ são relativamente pequenas [23, 24]. Esta informação adicional é, geralmente, expressa na forma:

$$
\mathcal{D}\left(\mathbf{R}_{k} \mathbf{f}_{k}\right)=P \beta_{R}
$$

onde $\mathcal{D}(\mathbf{x})$ é uma norma escolhida e $P$ é a dimensão do vetor $\mathbf{x}$. Neste trabalho são consideradas duas normas: a norma $\ell_{2}$, Euclidiana, ao quadrado e a norma Huber [25]. A matriz $\mathbf{R}_{k}$, de tamanho $P \times M$, representa um operador "passa-alta", como os operadores de diferenças finitas ou Laplaciano discreto. O $\beta_{R}$ é uma medida conhecida de $\mathcal{D}(\mathbf{x}) / P$. O vetor resultante de $\mathbf{R}_{k} \mathbf{f}_{k}$ representa as variações na intensidade de $\mathbf{f}_{k}$. Em muitos casos, o mesmo operador $\mathbf{R}_{k}$ é considerado para todas imagens da seqüência.

A norma $\ell_{2}$ ao quadrado, onde $\mathcal{D}(\mathbf{x})=\|\mathbf{x}\|_{2}^{2}=\sum_{i}\left|x_{i}\right|^{2}$, é geralmente escolhida porque garante a produção de imagens suaves e porque facilita a otimização dos algoritmos. Entretanto, esta norma pode produzir imagens exageradamente suaves. Muitos trabalhos [24, 25, 26] mencionam a necessidade de produção de imagens com bordas nítidas. Neste caso, a norma Huber pode substituir a norma $\ell_{2}$ visando preservar detalhes e bordas. A norma Huber é descrita por $\mathcal{D}(\mathbf{x})=\mathcal{H}_{T}(\mathbf{x})=\sum_{i} H_{T}\left(x_{i}\right)$, onde:

$$
H_{T}\left(x_{i}\right)=\left\{\begin{array}{cc}
\left|x_{i}\right|^{2} & \text { se }\left|x_{i}\right| \leq T \\
2 T\left|x_{i}\right|-T^{2} & \text { se }\left|x_{i}\right|>T
\end{array}\right.
$$

sendo $x_{i}$ um elemento do vetor $\mathbf{x}$. O parâmetro $T$ determina a influência da região quadrática ou da região linear da norma Huber. Neste modelo, a norma Huber preserva grandes valores das variações de amplitude de $\mathbf{f}_{k}$, gerando imagens com detalhes e bordas mais nítidas em relação à implementações baseadas na norma $\ell_{2}$.

O modelo de imagem, com a especificação de $\mathbf{R}_{k}$, de $\beta_{R}$ e da norma $\mathcal{D}(\mathbf{x})$, descreve a informação adicional necessária para ser utilizada na busca da imagem de alta resolução.

\footnotetext{
${ }^{2}$ Um problema mal-posto (ill-posed) é um problema matemático que tem, pelo menos, uma das seguintes características: não tem solução; tem infinitas soluções; ou a solução é instável em relação a entrada [19, 20].
} 


\section{ALGORITMOS EXISTENTES DE SUPER-RESOLUÇÃO}

Nesta análise comparativa serão considerados os algoritmos regularizados de super-resolução propostos em [1, 2, 4]. Os métodos regularizados são baseados na minimização de uma função de custo composta de um termo associado ao erro resultante entre as imagens capturadas e as imagens estimadas pelo algoritmo e de outro termo que visa a regularização do problema. A equação generalizada é:

$$
\hat{\boldsymbol{f}}=\arg \min _{\boldsymbol{f}} \overbrace{J(\boldsymbol{g}, \boldsymbol{f})}^{\text {Termo de dados }}+\overbrace{\lambda Q(\boldsymbol{f})}^{\text {Termo a priori }}
$$

onde a matriz $f$ representa a seqüência de imagens estimadas de alta resolução, e a matriz $\boldsymbol{g}$ representa os dados capturados, ou seja, a seqüência de baixa resolução. O termo $J(\boldsymbol{g}, \boldsymbol{f})$, chamado de termo de dados, fornece uma métrica de erro entre as imagens capturadas e as imagens estimadas. O termo $Q(\boldsymbol{f})$, chamado de termo de regularização ou de informação a priori, pondera a função de custo visando a regularização das estimativas e assim garantir a unicidade e a estabilidade da solução do problema. O escalar $\lambda$ é o coeficiente de regularização, o qual pode ser ajustado para controlar a influência relativa da informação a priori na função de custo.

Nesta seção são apresentados três algoritmos regularizados de super-resolução, em suas versões generalizadas. Estes algoritmos diferem na especificação das funções usadas nos termos de dados e no termo de informação a priori. Esta comparação dos algoritmos existentes servirão de suporte para, nas próximas seções, a discussão das características superiores da classe de algoritmos simultâneos que está sendo proposta.

\subsection{SUPER-RESOLUÇÃO DE ÚNICA IMAGEM}

Estes algoritmos baseiam-se na utilização de uma única imagem LR da seqüência por vez, a imagem temporalmente coincidente, $\mathbf{g}_{k}$, para a produção de uma única imagem $\mathrm{HR}$, $\hat{\mathbf{f}}_{k}$. A informação contida nas outras imagens LR da sequiência, $\mathbf{g}_{j}, j \neq k$, não são utilizadas para recuperar os detalhes da imagem escolhida da seqüência. Esta abordagem reduz as distorções causadas pela aquisição, produzindo uma imagem com uma qualidade maior do que a produzida por simples interpolação [27, 28].

A formulação geral desta abordagem é dada por:

$$
\hat{\mathbf{f}}_{k}=\arg \min _{\mathbf{f}_{k}}\left\|\mathbf{g}_{k}-\mathbf{D}_{k} \mathbf{f}_{k}\right\|_{2}^{2}+\lambda \mathcal{D}\left(\mathbf{R}_{k} \mathbf{f}_{k}\right)
$$

onde, segundo a estimativa Bayesiana [12], $\lambda$ é especificado como $\sigma_{\eta}^{2} / \beta_{R}$, sendo $\sigma_{\eta}^{2}$ a variância do ruído de aquisição e $\beta_{R}$ a medida da regularização, definida na seção 2.4 .

$\mathrm{O}$ algoritmo pode ser aplicado individualmente nas outras imagens da seqüência, para $k=1, \ldots, L$. A utilização da norma $\ell_{2}$ ao quadrado no termo de dados é justificada assumindo-se que o erro no termo de dados, o ruído $\eta$, pode ser modelado como aditivo, branco e Gaussiano [12, 23], conforme modelado na equação (1). Algoritmos desta classe são encontrados em [29, 30].

\subsection{SUPER-RESOLUÇÃO TRADICIONAL}

Os algoritmos tradicionais de super-resolução [1, 2, 18] utilizam toda a sequiência de imagens LR para a produção de uma imagem de HR. Em geral, aproveitando a informação proveniente das várias imagens da seqüência LR se obtém uma recuperação de detalhes aprimorada em relação aos algoritmos que se baseiam em uma única imagem LR isoladamente.

A formulação geral dos métodos tradicionais é descrita como:

$$
\hat{\mathbf{f}}_{k}=\arg \min _{\mathbf{f}_{k}} \sum_{j=1}^{k} \alpha_{j, k}\left\|\mathbf{g}_{j}-\mathbf{C}_{j, k} \mathbf{f}_{k}\right\|_{2}^{2}+\lambda \mathcal{D}\left(\mathbf{R}_{k} \mathbf{f}_{k}\right)
$$

onde $\alpha_{j, k}$ é uma ponderação aplicada em cada equação devido à presença do erro de movimento [18], esta ponderação é proporcional a razão da variância do ruído pela variância do erro no modelo combinado, ou seja, $\sigma_{\boldsymbol{\eta}}^{2} / \sigma_{\varepsilon_{j, k}}^{2}$. O escalar $\lambda$ é o mesmo definido na seção anterior. $\mathrm{O}$ algoritmo é aplicado individualmente para recuperar cada imagem HR da seqüência, para $k=1, \ldots, L$.

Nestes algoritmos, o movimento é utilizado no termo de dados, através do modelo combinado. Este termo proporciona a utilização da informação contida nas várias imagens da sequiência LR para recuperar os detalhes da imagem HR, $\mathbf{f}_{k}$.

\subsection{SUPER-RESOLUÇÃO SIMULTÂNEA}

Os algoritmos simultâneos estimam toda a seqüência de imagens HR conjuntamente, em um único processo. Esta abordagem permite a inclusão da informação sobre o movimento no termo de informação a priori. No termo a priori, a informação sobre o movimento é melhor aproveitada, proporcionando um ganho significativo na qualidade das imagens estimadas. Este ganho será explicitado e justificado na Seção 5.1. O primeiro trabalho sobre a abordagem simultânea foi proposto em [10], onde a equação de minimização é:

$$
\begin{gathered}
\hat{\mathbf{f}}_{1}, \ldots, \hat{\mathbf{f}}_{L}=\arg \min _{\mathbf{f}_{1}, \ldots, \mathbf{f}_{L}} \sum_{k=1}^{L} \sum_{j=1}^{L} \alpha_{j, k}\left\|\mathbf{g}_{j}-\mathbf{C}_{j, k} \mathbf{f}_{k}\right\|_{2}^{2}+ \\
\lambda\left[\sum_{k=1}^{L} \mathcal{H}\left(\mathbf{R}_{k} \mathbf{f}_{k}\right)+\right. \\
\left.\sum_{k=2}^{L-1} \mathcal{H}\left(-\mathbf{M}_{k, k-1} \mathbf{f}_{k-1}+2 \mathbf{f}_{k}-\mathbf{M}_{k, k+1} \mathbf{f}_{k+1}\right)\right]
\end{gathered}
$$

Em [10] é utilizado um operador de diferença finita de segunda ordem para modelar a matriz $\mathbf{R}_{k}$. Além disso foi escolhido um modelo de movimento de segunda ordem para o movimento no termo a priori. O trabalho em [10] considera apenas a norma Huber, $\mathcal{D}(\mathbf{x})=\mathcal{H}(\mathbf{x})$, e a minimização é realizada através do método de Gradiente Descente [19, 31]. Todavia, para as comparações neste trabalho é realizada a investigação da seguinte versão generalizada: 


$$
\begin{gathered}
\hat{\mathbf{f}}_{1}, \ldots, \hat{\mathbf{f}}_{L}=\arg \min _{\mathbf{f}_{1}, \ldots, \mathbf{f}_{L}} \sum_{k=1}^{L} \sum_{j=1}^{L} \alpha_{j, k}\left\|\mathbf{g}_{j}-\mathbf{C}_{j, k} \mathbf{f}_{k}\right\|_{2}^{2}+ \\
\lambda_{R} \sum_{k=1}^{L} \mathcal{D}\left(\mathbf{R}_{k} \mathbf{f}_{k}\right)+ \\
\lambda_{M} \sum_{k=2}^{L-1} \mathcal{D}\left(-\mathbf{M}_{k, k-1} \mathbf{f}_{k-1}+2 \mathbf{f}_{k}-\mathbf{M}_{k, k+1} \mathbf{f}_{k+1}\right)
\end{gathered}
$$

considerando diferença finita de segunda ordem nos modelos de imagem e de movimento, ou ainda:

$$
\begin{gathered}
\hat{\mathbf{f}}_{1}, \ldots, \hat{\mathbf{f}}_{L}=\arg \min _{\mathbf{f}_{1}, \ldots, \mathbf{f}_{L}} \sum_{k=1}^{L} \sum_{j=1}^{L} \alpha_{j, k}\left\|\mathbf{g}_{j}-\mathbf{C}_{j, k} \mathbf{f}_{k}\right\|_{2}^{2}+ \\
\lambda_{R} \sum_{k=1}^{L} \mathcal{D}\left(\mathbf{R}_{k} \mathbf{f}_{k}\right)+\lambda_{M} \sum_{k=2}^{L} \mathcal{D}\left(\mathbf{f}_{k}-\mathbf{M}_{k, k-1} \mathbf{f}_{k-1}\right)
\end{gathered}
$$

considerando diferença finita de primeira ordem nos modelos. A generalização é constituída pela utilização de parâmetros de regularização independentes para o termo de diferença finita espacial e de diferença finita na trajetória de movimento. Além disso, são consideradas as normas Euclidiana e Huber em $\mathcal{D}(\mathbf{x})$ e os modelos de primeira e segunda ordem.

\section{SUPER-RESOLUÇÃO SIMULTÂNEA PROPOSTA}

Nesta seção é descrito o novo algoritmo simultâneo proposto, que difere dos demais por explorar a utilização do movimento apenas no termo de informação a priori. Como resultado, o método proposto requer uma quantidade de equações menor no termo de dados quando comparado com [10]. A seguir, na Seção 5.1, é oferecida uma análise das diversas vantagens da utilização do movimento somente no termo a priori.

\subsection{MODELO GERAL PROPOSTO}

A formulação mais simples, descrita abaixo, utiliza modelo de movimento de primeira ordem:

$$
\begin{aligned}
& \hat{\mathbf{f}}_{1}, \ldots, \hat{\mathbf{f}}_{L}=\arg \min _{\mathbf{f}_{1}, \cdots, \mathbf{f}_{L}} \sum_{k=1}^{L}\left\|\mathbf{g}_{k}-\mathbf{D}_{k} \mathbf{f}_{k}\right\|_{2}^{2}+ \\
& \lambda_{R} \sum_{k=1}^{L} \mathcal{D}\left(\mathbf{R}_{k} \mathbf{f}_{k}\right)+\lambda_{M} \sum_{k=2}^{L} \mathcal{D}\left(\mathbf{f}_{k-1}-\mathbf{M}_{k-1, k} \mathbf{f}_{k}\right)
\end{aligned}
$$

são utilizados os seguintes valores para os coeficientes de regularização: $\lambda_{R}=\sigma_{\boldsymbol{\eta}}^{2} / \beta_{R}$ e $\lambda_{R}=\sigma_{\boldsymbol{\eta}}^{2} / \beta_{M}$, sendo que $\beta_{M}$ é uma medida do erro de movimento $\mathcal{D}\left(\mathbf{f}_{k-1}-\mathbf{M}_{k-1, k} \mathbf{f}_{k}\right) / M$ assumida igual para todos os quadros para reduzir o número de coeficientes. Estes coeficientes são os coeficientes recomendados pela estimativa estatística Bayesiana $[12,19]$.

Pode-se observar uma grande redução no número de equações no termo de dados em (14) quando comparado com 50
(11). Nesta proposta são retiradas do termo de dados as expressões compostas pelo modelo combinado, reduzindo de $L^{2}$ para $L$ equações neste termo. Além disso, é possível considerar modelos de diferentes ordens no termo de informação a priori, desde modelos simples como o modelo de primeira ordem até modelos de ordem maior.

A redução do número de termos nas equações em (14), normalmente não ocasiona perdas de qualidade devido à recursividade do algoritmo. A minimização da diferença entre quadros adjacentes, no termo de informação a priori, proporciona a propagação da informação necessária para superresolução entre os quadros da seqüência de forma recursiva. Por exemplo, o processo de otimização visa a minimização da função de custo forçando com que $\mathbf{f}_{1}$ se aproxime de $\mathbf{M}_{1,2} \mathbf{f}_{2}$ e $\mathbf{f}_{2}$ se aproxime de $\mathbf{M}_{2,3} \mathbf{f}_{3}$, logo, também proporciona uma propagação entre quadros tal que $\mathbf{f}_{1}$ se aproxime de $\mathbf{M}_{1,2} \mathbf{M}_{2,3} \mathbf{f}_{3}=\mathbf{M}_{1,3} \mathbf{f}_{3}$. Desta forma cada imagem da seqüência estará contribuindo para a reconstrução dos detalhes em todas as outras. Esta é uma característica exclusiva dos métodos simultâneos.

A forma generalizada do método proposto é:

$$
\begin{aligned}
& \hat{\boldsymbol{f}}=\left[\hat{\mathbf{f}}_{1}^{T} \cdots \hat{\mathbf{f}}_{L}^{T}\right]^{T}=\arg \min _{\boldsymbol{f}}\|\boldsymbol{g}-\boldsymbol{D} \boldsymbol{f}\|_{2}^{2}+ \\
& \lambda_{R} \mathcal{D}(\boldsymbol{R} \boldsymbol{f})+\lambda_{M} \mathcal{D}(\boldsymbol{M} \boldsymbol{f})
\end{aligned}
$$

onde $\boldsymbol{g}=\left[\mathbf{g}_{1}^{T} \cdots \mathbf{g}_{L}^{T}\right]^{T}$ é a sequiência LR e $\boldsymbol{f}=\left[\mathbf{f}_{1}^{T} \cdots \mathbf{f}_{L}^{T}\right]^{T}$ é a sequiência HR. As matrizes em (15) são:

$$
\boldsymbol{D}=\left[\begin{array}{ccc}
\mathbf{D}_{1} & \cdots & \mathbf{0} \\
\vdots & \ddots & \vdots \\
\mathbf{0} & \cdots & \mathbf{D}_{L}
\end{array}\right]
$$

de tamanho $L N \times L M$, para o modelo de aquisição,

$$
\boldsymbol{R}=\left[\begin{array}{ccc}
\mathbf{R}_{1} & \cdots & \mathbf{0} \\
\vdots & \ddots & \vdots \\
\mathbf{0} & \cdots & \mathbf{R}_{L}
\end{array}\right]
$$

de tamanho $L P \times L M$, para o modelo de imagem, e

$$
\boldsymbol{M}=\left[\begin{array}{cccc}
-\mathbf{M}_{2,1} & \mathbf{I} & \cdots & \mathbf{0} \\
\vdots & \ddots & \ddots & \vdots \\
\mathbf{0} & \cdots & -\mathbf{M}_{L, L-1} & \mathbf{I}
\end{array}\right]
$$

de tamanho $(L-1) M \times L M$, para o modelo de movimento de primeira ordem, onde $\mathbf{I}$ é a matriz identidade. A matriz usada para representar o modelo de segunda ordem é:

$$
\boldsymbol{M}=\left[\begin{array}{ccccc}
-\mathbf{M}_{2,1} & 2 \mathbf{I} & -\mathbf{M}_{2,3} & \cdots & \mathbf{0} \\
\vdots & \ddots & \ddots & \ddots & \vdots \\
\mathbf{0} & \cdots & -\mathbf{M}_{L-1, L-2} & 2 \mathbf{I} & -\mathbf{M}_{L-1, L}
\end{array}\right]
$$

de tamanho $(L-2) M \times L M$.

\subsection{OTIMIZAÇÃO}

As funções de custo apresentadas em (14) e (15) podem ser minimizadas através de métodos baseados no gradiente. 
Neste trabalho são utilizados os métodos de Gradiente Conjugado (GC) Linear, para os casos onde $\mathcal{D}(\mathbf{x})=\|\mathbf{x}\|_{2}^{2}$, e o Gradiente Conjugado Não-Linear proposto por Fletcher e Reeves [19, 31, 32], para os casos onde $\mathcal{D}(\mathbf{x})=\mathcal{H}(\mathbf{x})$. O método do Gradiente Conjugado é significativamente mais rápido do que o método do Gradiente Descente, o qual é utilizado em [10].

No método GC Linear é necessário calcular a matriz $A=$ $\boldsymbol{D}^{T} \boldsymbol{D}+\lambda_{R} \boldsymbol{R}^{T} \boldsymbol{R}+\lambda_{M} \boldsymbol{M}^{T} \boldsymbol{M}$ e o vetor $\boldsymbol{b}=\boldsymbol{D}^{T} \boldsymbol{g}$. Já no método GC Não-Linear é necessário utilizar o seguinte gradiente:

$$
\begin{aligned}
\frac{1}{2} \nabla J(\boldsymbol{f})=-\boldsymbol{D}^{T}(\boldsymbol{g}-\boldsymbol{D} \boldsymbol{f})+ \\
\lambda_{R} \boldsymbol{R}^{T} \mathcal{T}_{T}(\boldsymbol{R} \boldsymbol{f})+\lambda_{M} \boldsymbol{M}^{T} \mathcal{T}_{T}(\boldsymbol{M} \boldsymbol{f})
\end{aligned}
$$

onde $\mathcal{T}_{T}(\boldsymbol{x})=\left[T\left(x_{1}\right), \cdots, T_{T}\left(x_{q}\right)\right]^{T}$ e:

$$
T_{T}(x)= \begin{cases}x, & \text { se }|x| \leq T \\ T \operatorname{sign}(x), & \text { se }|x|>T\end{cases}
$$

Na busca unidimensional do GC é utilizado o algoritmo da Secante [32].

\section{COMPARAÇÕES}

\subsection{QUALIDADE OBTIDA PELOS MÉTODOS SIMULTÂNEOS}

O método simultâneo proposto neste trabalho, assim como o método em [10], utiliza uma função de restrição para penalizar o erro de movimento em alta resolução. Esta penalidade propicia uma qualidade superior em relação aos métodos tradicionais. $\mathrm{O}$ erro de movimento é minimizado em alta resolução, no termo a priori, permitindo resultados mais consistentes com o modelo de movimento da equação (2). As funções de restrições utilizadas são do tipo:

$$
\left\|\mathbf{f}_{k-1}-\mathbf{M}_{k-1, k} \mathbf{f}_{k}\right\|=\left\|\mathbf{e}_{k-1, k}\right\|
$$

onde esta restrição leva a uma minimização do erro no modelo de movimento $\mathbf{e}_{k-1, k}$ em alta resolução.

Entretanto, nos métodos tradicionais, que usam o modelo combinado, o erro de movimento é minimizado em baixa resolução, no termo de dados:

$$
\begin{gathered}
\left\|\mathbf{g}_{k}-\mathbf{C}_{k, j} \mathbf{f}_{j}\right\|=\left\|\varepsilon_{k, j}\right\| \\
\left\|\mathbf{g}_{k}-\mathbf{D}_{k} \mathbf{M}_{k, j} \mathbf{f}_{j}\right\|=\left\|\boldsymbol{\varepsilon}_{k, j}\right\| \\
\left\|\mathbf{D}_{k} \mathbf{f}_{k}+\boldsymbol{\eta}_{k}-\mathbf{D}_{k} \mathbf{M}_{k, j} \mathbf{f}_{j}\right\|=\left\|\boldsymbol{\epsilon}_{k, j}+\boldsymbol{\eta}_{k}\right\| \\
\left\|\mathbf{D}_{k}\left(\mathbf{f}_{k}-\mathbf{M}_{k, j} \mathbf{f}_{j}\right)+\boldsymbol{\eta}_{k}\right\|=\left\|\mathbf{D}_{k} \mathbf{e}_{k, j}+\boldsymbol{\eta}_{k}\right\|
\end{gathered}
$$

A presença do operador $\mathbf{D}_{k}$, o qual representa o mapeamento de HR para LR, inclui a dizimação e outros efeitos. Este operador gera perdas e distorções na informação da diferença entre as imagens estimadas HR. Assim, diferentemente dos métodos simultâneos, os métodos tradicionais de super-resolução não oferecem uma solução consistente com o modelo de movimento de (2). Portanto, os métodos simultâneos, os quais minimizam o erro de movimento em alta resolução, oferecem uma seqüência de imagens resultante com maior suavidade ao longo da trajetória de movimento.

\subsection{REDUÇÃO DE CUSTO COMPUTACIONAL}

Uma das principais contribuições deste trabalho é a redução computacional obtida em relação ao método simultâneo proposto em [10]. A redução de custo computacional é obtida através da redução do número de termos na função de custo e através de otimização eficiente baseada nos métodos de Gradiente Conjugado.

Nos algoritmos com penalidade $\ell_{2}$ ocorre uma redução na complexidade computacional pois é necessário um número menor de operações para a solução do problema. Conseqüentemente, a matriz $\boldsymbol{A}$ e o vetor $\boldsymbol{b}$, definidos na Seção $4.2 \mathrm{e}$ necessários no método do Gradiente Conjugado Linear, são obtidos mais rapidamente levando a um tempo menor de iniciação do processo iterativo. Além disso, a matriz $\boldsymbol{A}$ resultante é mais esparsa, fazendo com que o cálculo por iteração seja também menor.

A Tabela 1 apresenta o custo computacional por iteração requerida pelo GC para os métodos apresentados, utilizando modelos de primeira ordem e armazenamento esparso. Nesta tabela é apresentado o número de operações, onde o custo de uma multiplicação é assumido igual ao de uma adição. Observe que nos métodos simultâneos uma iteração atualiza as $L$ imagens da seqüência, enquanto que no método de única imagem e no método tradicional cada iteração atualiza uma imagem da seqüencia, requerendo $L$ iterações para atualizar as $L$ imagens da sequiência.

Tabela 1. Custo por iteração do GC em operações (adição ou multiplicação)

\begin{tabular}{|l||l|}
\hline Algoritmo SR & Custo por iteração para $L$ imagens \\
\hline \hline Única Imagem & $L(10 M+4(D N+B P))$ \\
\hline Tradicional & $L(10 M+4(D L N+B P))$ \\
\hline Simult. de [10] & $10 L M+4\left(D L^{2} N+B L P+2(L-1) M\right)$ \\
\hline Simult. Proposta & $10 L M+4(D L N+B L P+2(L-1) M)$ \\
\hline
\end{tabular}

Sendo:

$L$ - Número de imagens na seqüência

$M \quad$ - Número de pixels na imagem HR

$N \quad$ - Número de pixels na imagem LR

$P \quad$ - Dimensão do vetor resultante de $\mathbf{R}_{k} \mathbf{f}_{k}$

$B$ - Número de elementos não-nulos por linha em $\mathbf{R}_{k}$

$D \quad$ - Número de elem. não-nulos por linha em $\mathbf{D}_{k}$ ou $\mathbf{C}_{k, j}$

Na Tabela 2 é apresentado um exemplo com valores tipicamente encontrados nos problemas de super-resolução. Estes valores correspondem aos valores encontrados nos experimentos realizados na Seção 6. Observe que, o custo computacional por iteração é proporcional a $L^{2}$ no método tradicional e no método simultâneo proposto em [10]. Por outro lado, no método de única imagem e no método simultâneo proposto o custo por iteração é proporcional a $L$.

Tabela 2. Exemplo de custo por iteração do GC com valores típicos encontrados em SR

\begin{tabular}{|l||l|}
\hline Algoritmo SR & Custo por iteração neste exemplo \\
\hline \hline Única Imagem & $(40+64+128) L N$ \\
\hline Tradicional & $(40+64 L+128) L N$ \\
\hline Simultânea de [10] & $\left(40+64 L+128+32 \frac{L-1}{L}\right) L N$ \\
\hline Simultânea Proposta & $\left(40+64+128+32 \frac{L-1}{L}\right) L N$ \\
\hline
\end{tabular}




\section{Marcelo Victor Wüst Zibetti e Joceli Mayer Super-Resolução Regularizada e Simultânea de Seqüência de Imagens}

Onde os valores típicos de SR: $D=16, B=2, P=4 M$, $M=4 N$.

Em geral, o número de iterações necessárias para a convergência do GC é independente do método de superresolução utilizado, contudo, é dependente do condicionamento das matrizes envolvidas.

Nos algoritmos com penalidade Huber o processo de otimização é não-linear. Em conseqüência, a redução no número de equações do método proposto tem um impacto menor na redução de custo computacional. Em geral, utilizando o método de Gradiente Conjugado Não-Linear, obtém-se um desempenho superior ao do método simultâneo proposto em [10]. O Gradiente Conjugado Não-Linear possui uma velocidade de convergência maior do que a do método de Gradiente Descente utilizado em [10].

\subsection{ROBUSTEZ DO MÉTODO PROPOSTO}

A ocorrência de outliers na seqüência de imagens reduz a qualidade dos resultados produzidos pelos algoritmos de super-resolução que utilizam o movimento. Desta forma, é necessário a utilização de métodos robustos e/ou de procedimentos de detecção e eliminação de outliers.

Entre os algoritmos revisados neste trabalho, apenas os métodos que utilizam uma única imagem são imunes aos outliers porque não utilizam o movimento em suas equações. Todos os demais, que utilizam o modelo de movimento, estão suscetíveis aos problemas ocasionados pelos outliers.

A norma $\ell_{2}$ ao quadrado, associada às equações de movimento, reduz a robustez dos algoritmos aos outliers, pois os grandes erros, ou outliers, tem grande influência na estimativa e causam grandes desvios, ou distorções, no resultado. Para evitar estas distorções é necessário um procedimento de detecção e eliminação de outliers como proposto em $[17,15,33]$.

Para os métodos tradicionais, os outliers afetam as expressões que envolvem o modelo combinado no termo de dados. Portanto, é necessário a remoção destes, conforme mencionado na Seção 2.3, no termo de dados para evitar distorções na estimativa. No método simultâneo proposto em [10], os outliers afetam tanto as expressões do modelo combinado, no termo de dados, quanto expressões que envolvem o movimento no termo a priori. Portanto, é necessária sua remoção nos dois termos. Já no algoritmo proposto, os outliers afetam apenas o termo a priori, onde as expressões de movimento estão alocadas. Portanto, sua remoção, conforme especificado na Seção 2.2, é necessária apenas no termo $a$ priori.

Uma grande vantagem do método proposto é a robustez do algoritmo aos outliers quando a norma Huber é utilizada no termo a priori. Nos métodos tradicionais e de única imagem esta norma é utilizada no termo de regularização associada aos operadores de diferença finita espacial, com o intuito de preservar as bordas dos objetos nas imagens. A preservação das bordas ocorre porque a norma Huber considera linearmente os grandes erros e quadraticamente os pequenos erros. Contudo, neste trabalho foi observado que a norma Huber, quando associada ao operadores de movimento no termo $a$ priori, torna o algoritmo menos sensível a influência degen- erativa dos outliers existentes no movimento. Os algoritmos tradicionais e o simultâneo proposto em [10] utilizam o movimento no termo de dados, associado a norma $\ell_{2}$ ao quadrado, e desta forma não exploram as vantagens da norma Huber, como o método proposto. Portanto, o algoritmo proposto utilizando a norma Huber apresenta maior robustez aos outliers do que esses algoritmos, como será observado nos experimentos.

\section{EXPERIMENTOS}

Para ilustrar o desempenho dos algoritmos foi realizado o seguinte experimento: foi simulada a aquisição de quatro seqüências de imagens conhecidas. Estas seqüências são mostradas nas figuras 3 até 6 , com as respectivas dimensões de captura. Pode-se observar visualmente as distorções causadas pela aquisição com baixa resolução. A função de aquisição considerada envolve distorção óptica e subamostragem de duas vezes nas direções horizontal e vertical. A variância do ruído aditivo é proporcional a variância da imagem, resultando em $\mathrm{SNR}=40 \mathrm{~dB}$.

Assume-se que as seqüências de imagens das figuras 3 e 4 seguem um movimento global conhecido. Entretanto, existem objetos presentes nas seqüências que seguem um movimento diferente do assumido (o círculo preto na Figura 3 e o veículo na Figura 4). Estes objetos causam grandes erros na estimativa e são considerados outliers. Eles precisam ser detectados e removidos, caso contrário reduzirão significativamente o desempenho dos algoritmos não-robustos.

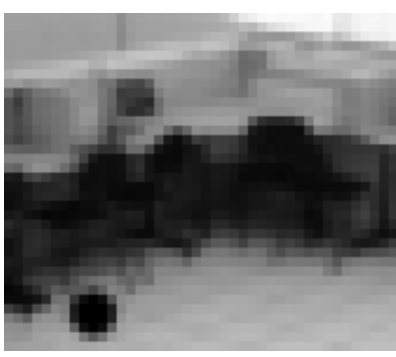

(a) Imagem 1

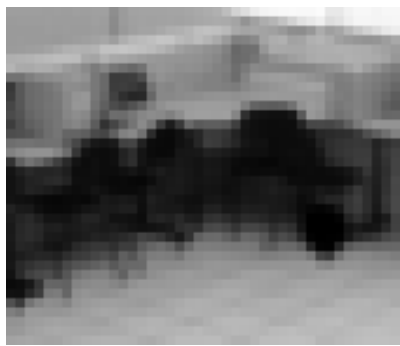

(c) Imagem 3

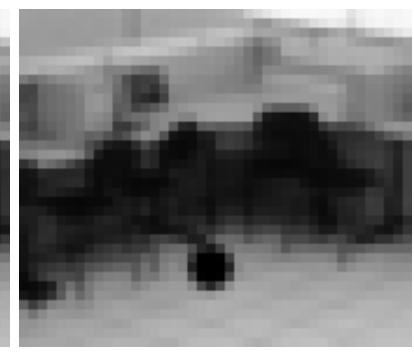

(b) Imagem 2

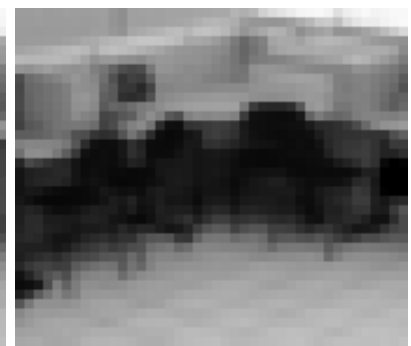

(d) Imagem 4
Figura 3. Seqüência Capturada Sala, dimensões $42 \times 36 \times 4$, movimento conhecido. 


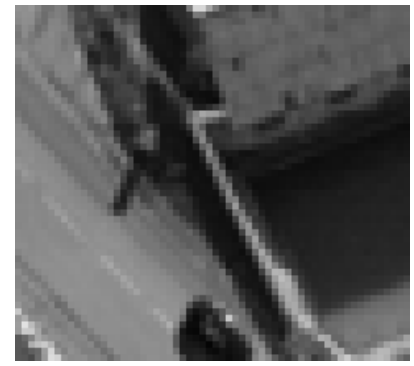

(a) Imagem 1

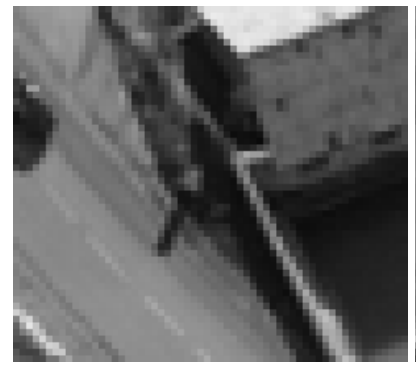

(c) Imagem 3

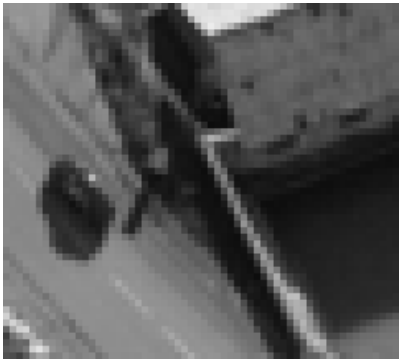

(b) Imagem 2

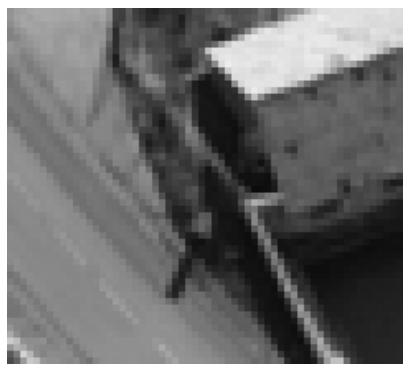

(d) Imagem 4
Figura 4. Seqüência Capturada Estrada, dimensões $60 \times$ $64 \times 4$, movimento conhecido.

Nas seqüências de imagens das figuras 5 e 6 o movimento foi assumido como independente em cada pixel, onde o campo de vetores que representa o movimento foi estimado com o algoritmo de fluxo óptico descrito em [34]. Nestas duas sequiências os outliers ocorrem em diversos lugares nas imagens, principalmente devido a oclusão causada pelo movimento dos objetos mais próximos da câmera (como o trem na sequiência da Figura 5 e a árvore na sequiência da Figura 6).

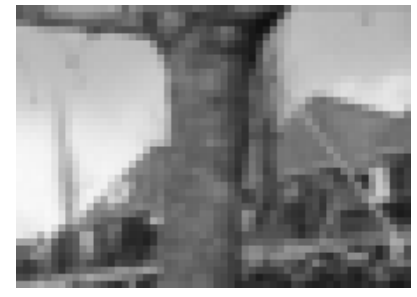

(a) Imagem 1

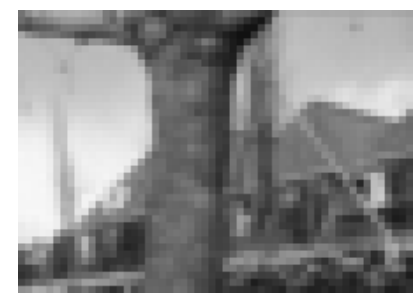

(c) Imagem 3

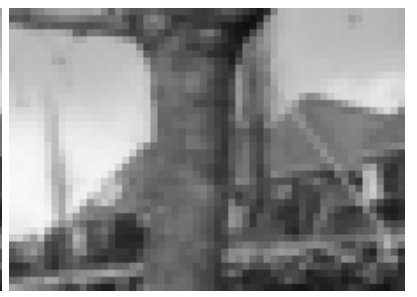

(b) Imagem 2

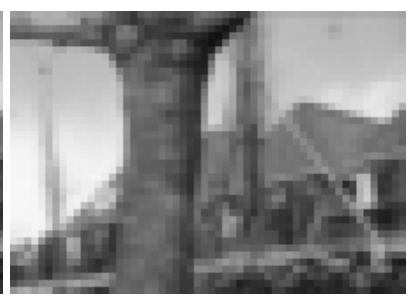

(d) Imagem 5
Figura 5. Sequiência Capturada Flower Garden, dimensões $56 \times 40 \times 5$, movimento estimado.

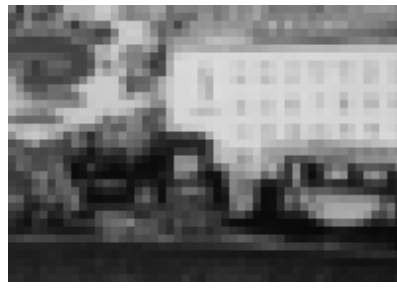

(a) Imagem 1

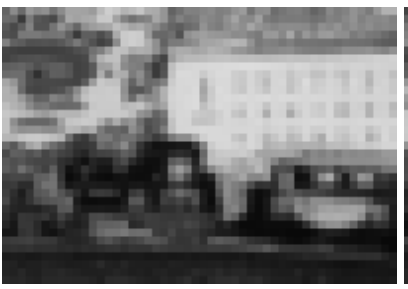

(c) Imagem 3

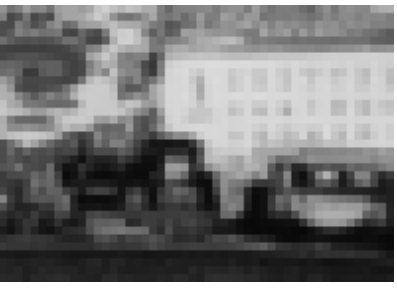

(b) Imagem 2

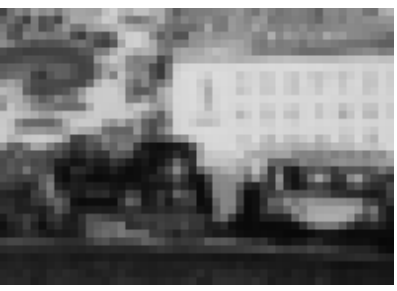

(d) Imagem 5
Figura 6. Seqüência Capturada Mobile Calendar, $50 \times 35 \times 5$, movimento estimado.

Foram detectados como outliers os pixels que apresentam valores absolutos de $\mathbf{e}_{k, j}^{*}=\mathbf{f}_{k}^{*}-\mathbf{M}_{k, j} \mathbf{f}_{j}^{*}$ e $\varepsilon_{k, j}^{*}=\mathbf{g}_{k}-$ $\mathbf{C}_{k, j} \mathbf{f}_{j}^{*}$ maiores que um limiar $\kappa$, definido como $\kappa=\mu+$ $2 \sigma$, onde $\mu$ é a média dos elementos do vetor $\mathbf{e}_{k, j}^{*}$ ou $\varepsilon_{k, j}^{*}$ e $\sigma$ é o desvio padrão dos elementos do vetor $\mathbf{e}_{k, j}^{*}$ ou $\varepsilon_{k, j}^{*}$, conforme sugerido em [15]. Neste trabalho foi utilizado $\mathbf{f}_{k}^{*}$ como uma imagem com interpolação linear de $\mathbf{g}_{k}$. Os pixels considerados outliers são removidos das equações, conforme a descrição apresentada nas seções 2.2 e 2.3.

As seqüências de imagens foram recuperadas, utilizando os seguintes algoritmos:

1. SR-UIM: O algoritmo de super-resolução de única imagem, apresentado na equação (9). Este algoritmo não explora a informação contida nas várias imagens da seqüência para obtenção da super-resolução. O movimento não é utilizado no termo de dados e no termo de informação a priori.

2. SR-TRA: O algoritmo tradicional de super-resolução, apresentado na equação (10). Este algoritmo utiliza o movimento no termo de dados, entretanto ele requer detecção e eliminação de outliers.

3. SR-BOR: A versão generalizada do algoritmo de superresolução simultânea proposto em [10], descrita nas equações (12) e (13). Este algoritmo utiliza o movimento no termo de dados e no termo de informação a priori. Este algoritmo também requer detecção e eliminação de outliers.

4. SR-SIM: O algoritmo de super-resolução simultânea proposto neste trabalho, apresentado na equação (15). Este algoritmo utiliza o movimento apenas no termo de informação a priori. O processo de detecção e eliminação de outliers é mais necessário no caso da norma $\ell_{2}$. No caso da norma Huber é esperada uma robustez maior, tornando o processo de detecção e eliminação de outliers praticamente desnecessário.

Os algoritmos foram comparados utilizando:

- A penalidade com norma $\ell_{2}, \mathcal{D}(\mathbf{x})=\|\mathbf{x}\|_{2}^{2}$, para obtenção de baixo custo computacional, e a penalidade com norma Huber, $\mathcal{D}(\mathbf{x})=\mathcal{H}(\mathbf{x})$, para obter um algoritmo que preserva as bordas dos objetos nas imagens estimadas e, no caso do algoritmo proposto, para obter 
robustez aos outliers no movimento.

- As matrizes $\mathbf{R}_{k}$ e $\boldsymbol{M}$ com modelos de primeira e segunda ordem.

- A detecção dos outliers ativada, para observar a melhor qualidade que pode ser obtida pelo algoritmo, e a detecção dos outliers desativada, para observar a robustez aos outliers do algoritmo.

- A minimização através do método de Gradiente Conjugado, sugerido neste trabalho, e a minimização através do método de Gradiente Descente, sugerido em [2, 10].

A qualidade objetiva das imagens estimadas foi avaliada em termos de PSNR [23]. Para observar a robustez aos outliers, são comparados o PSNR com a detecção dos outliers ativada e desativada. Os resultados numéricos, mostrando a média dos resultados de todas as seqüências, são apresentados nas tabelas 3 e 4 . Alguns resultados visuais são mostrados nas figuras 7 até 13. Para comparação do custo computacional são apresentados o tempo necessário até a convergência na Tabela 5. Nesta tabela é indicada a razão do tempo de convergência do método em relação ao tempo de convergência do algoritmo mais rápido, o método SR-UIM.

Tabela 3. Resultados médios de PSNR, em dB, para norma $\ell_{2}$ ao quadrado

\begin{tabular}{|c||c|c|c|c|}
\hline \multicolumn{1}{|c||}{} & \multicolumn{2}{c|}{ Detecção Ativada } & \multicolumn{2}{c|}{ Detecção Desativada } \\
\hline \hline Algoritmo & Ordem 1 & Ordem 2 & Ordem 1 & Ordem 2 \\
\hline \hline SR-UIM & 26.7 & 26.7 & 26.7 & 26.7 \\
\hline SR-TRA & 28.5 & 28.5 & 22.3 & 23.8 \\
\hline SR-BOR & 29.1 & 29.0 & 23.3 & 25.2 \\
\hline SR-SIM & $\mathbf{2 9 . 1}$ & $\mathbf{2 9 . 0}$ & 24.7 & 26.1 \\
\hline
\end{tabular}

Tabela 4. Resultados médios de PSNR, em dB, para norma Huber

\begin{tabular}{|c||c|c|c|c|}
\hline & \multicolumn{2}{c|}{ Detecção Ativada } & \multicolumn{2}{c|}{ Detecção Desativada } \\
\hline \hline Algoritmo & Ordem 1 & Ordem 2 & Ordem 1 & Ordem 2 \\
\hline \hline SR-UIM & 26.5 & 26.9 & 26.5 & 26.9 \\
\hline SR-TRA & 28.0 & 28.5 & 12.1 & 15.8 \\
\hline SR-BOR & 28.8 & 29.2 & 15.5 & 18.4 \\
\hline SR-SIM & $\mathbf{2 8 . 9}$ & $\mathbf{2 9 . 2}$ & $\mathbf{2 8 . 7}$ & $\mathbf{2 9 . 1}$ \\
\hline
\end{tabular}

Tabela 5. Tempo para convergência (Ordem 1/Ordem 2), normalizado

\begin{tabular}{|c||c|c|c|c|}
\hline \multicolumn{1}{|c||}{} & \multicolumn{2}{c|}{ Norma $\ell_{2}$} & \multicolumn{2}{c|}{ Norma Huber } \\
\hline \hline Algoritmo & G. Conj. & G. Desc. & G. Conj. & G. Desc. \\
\hline \hline SR-UIM & $1.0 / 1.1$ & $3.0 / 3.2$ & $9.1 / 8.1$ & $18.2 / 22.3$ \\
\hline SR-TRA & $2.4 / 2.5$ & $7.5 / 7.7$ & $17.9 / 19.1$ & $30.1 / 37.3$ \\
\hline SR-BOR & $4.5 / 4.6$ & $11.1 / 11.2$ & $22.4 / 24.7$ & $45.1 / 49.7$ \\
\hline SR-SIM & $\mathbf{2 . 2} / \mathbf{2 . 5}$ & $6.2 / 7.6$ & $\mathbf{1 6 . 7} / \mathbf{1 7 . 1}$ & $36.6 / 43.4$ \\
\hline
\end{tabular}

As tabelas 3 e 4 indicam que o método SR-SIM proporciona uma qualidade similar ao SR-BOR, com a detecção de outliers ativada. Os dois algoritmos simultâneos produzem resultados superiores aos métodos tradicionais, os quais, por sua vez, produzem resultados superiores aos métodos de única imagem. Observa-se que o algoritmo proposto em [10], cujos resultados são mostrados na Tabela 4 (com norma Huber, detecção ativada e ordem 2), e o algoritmo SR-SIM, com as mesmas especificações, obtiveram os melhores resultados. Nas figuras 7 e 8 são apresentados alguns resultados com a utilização da norma $\ell_{2}$. Nas figuras 9 e 10 são apresentados alguns resultados com a utilização da norma Huber.

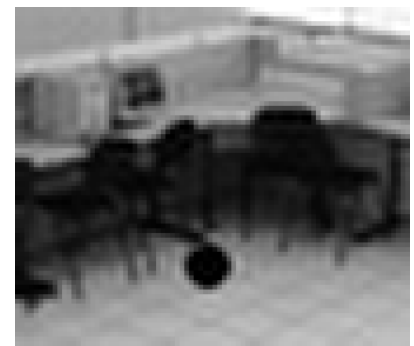

(a) SR-UIM (28.7 dB)

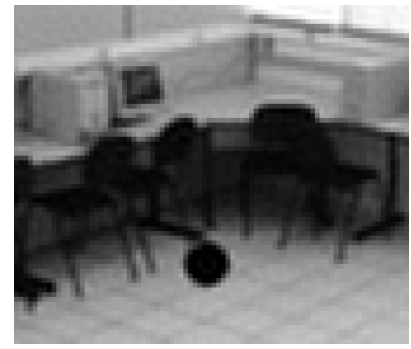

(c) SR-BOR $(32.0 \mathrm{~dB})$

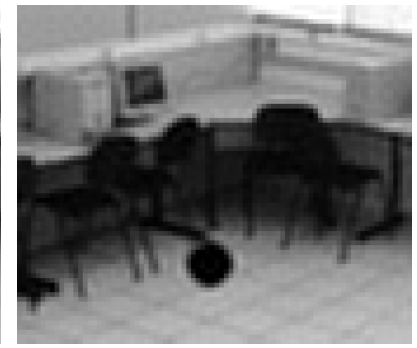

(b) SR-TRA $(31.6 \mathrm{~dB})$

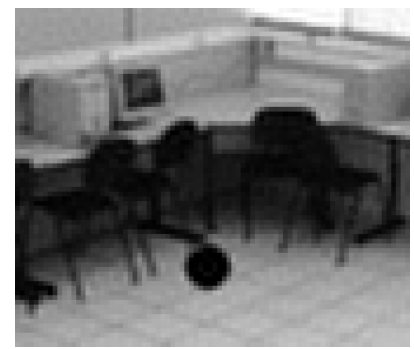

(d) SR-SIM (32.1 dB)
Figura 7. Resultados para a imagem 2 da seqüência Sala (movimento conhecido), com norma $\ell_{2}$, detecção de outliers ativada e modelos de segunda ordem.

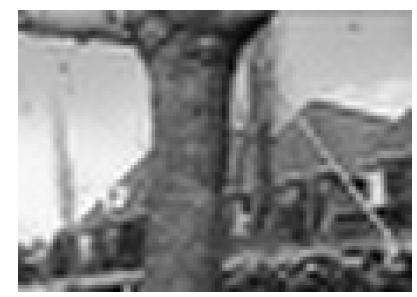

(a) SR-UIM (24.1 dB)

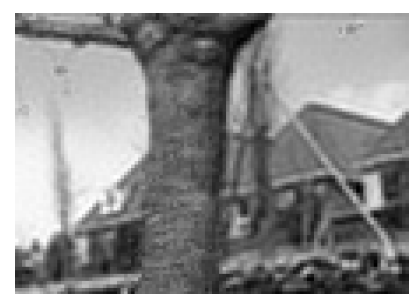

(c) SR-BOR $(26.0 \mathrm{~dB})$

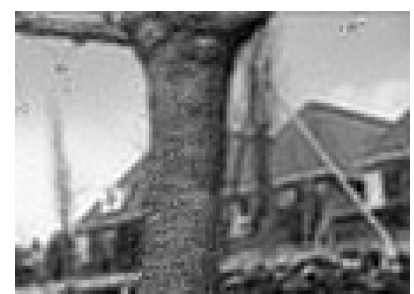

(b) SR-TRA $(25.5 \mathrm{~dB})$

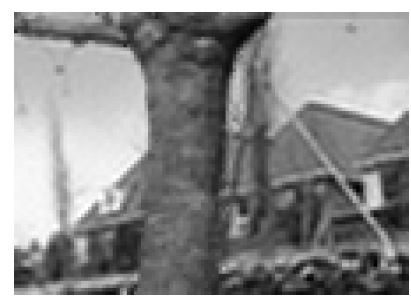

(d) SR-SIM (25.8 dB)
Figura 8. Resultados para a imagem 3 da sequiência Flower Garden (movimento estimado), com norma $\ell_{2}$, detecção de outliers ativada e modelos de primeira ordem. 


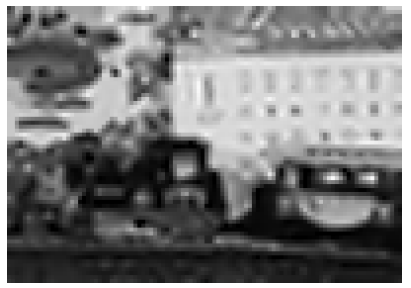

(a) SR-UIM (20.9 dB)

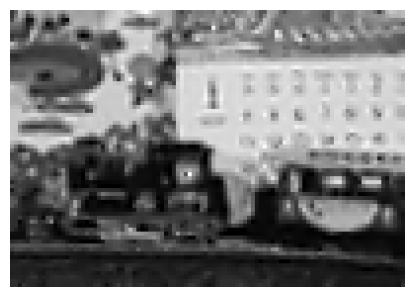

(c) SR-BOR (21.9 dB)

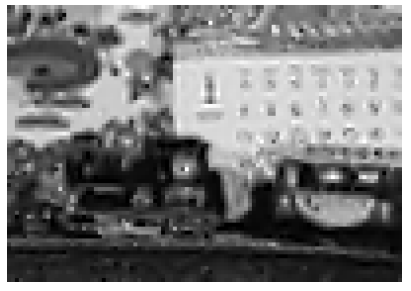

(b) SR-TRA (21.3 dB)

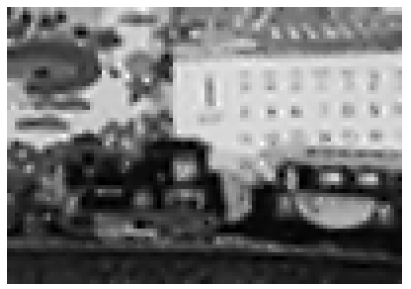

(d) SR-SIM (21.8 dB)
Figura 9. Resultados visuais da imagem 3 da sequiência Mobile Calendar (movimento estimado), com norma Huber, detecção de outliers ativada e modelos de segunda ordem.

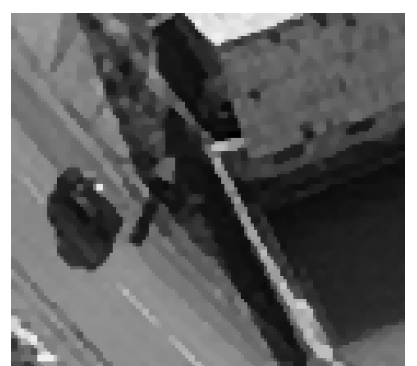

(a) SR-UIM (30.4 dB)

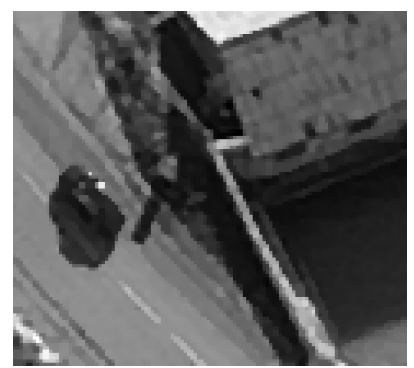

(c) SR-BOR (33.6 dB)

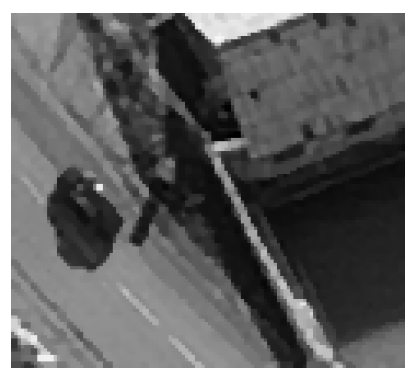

(b) SR-TRA (32.6 dB)

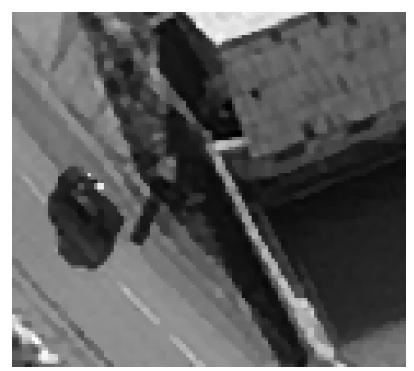

(d) SR-SIM (33.7 dB)
Figura 10. Resultados visuais da imagem 2 da seqüência Estrada (movimento conhecido), com norma Huber, detecção de outliers ativada e modelos de primeira ordem.

Constata-se também na Tabela 3 que os algoritmos com norma $\ell_{2}$ não são robustos aos outliers, pois com a detecção desativada a qualidade reduziu significativamente, inclusive apresentando qualidade inferior ao do algoritmo de única imagem. Nos resultados visuais das figuras 11 e 12, onde a detecção dos outliers foi desativada, pode-se observar que todos os algoritmos, com exceção do SR-UIM que não explora o movimento entre as imagens, produzem imagens com distorções nas regiões onde ocorrem os outliers.

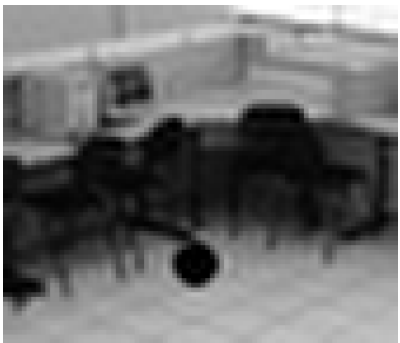

(a) SR-UIM (28.7 dB)

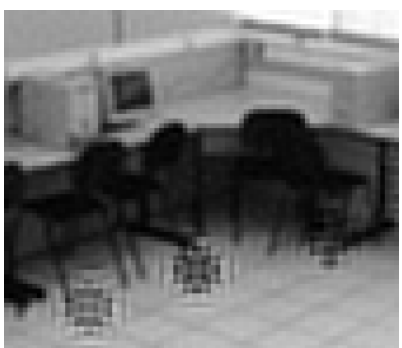

(c) SR-BOR $(26.0 \mathrm{~dB})$

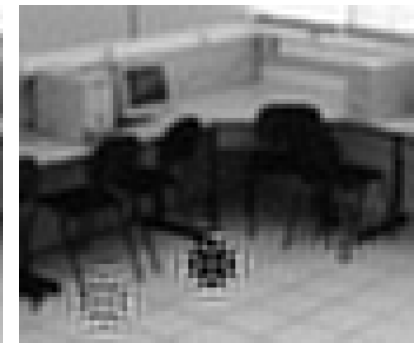

(b) SR-TRA $(27.5 \mathrm{~dB})$

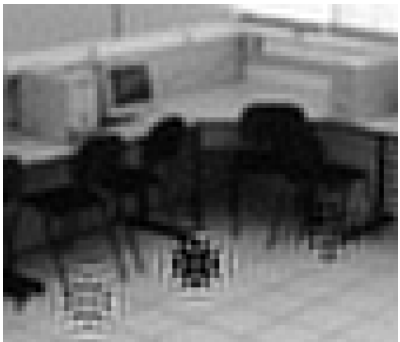

(d) SR-SIM (27.3 dB)
Figura 11. Resultados visuais da imagem 2 da seqüência Sala (movimento conhecido), com norma $\ell_{2}$, detecção de outliers desativada e modelos de segunda ordem.

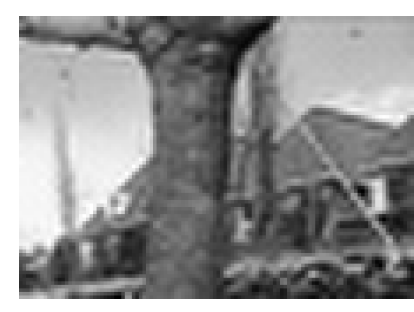

(a) SR-UIM (24.1 dB)

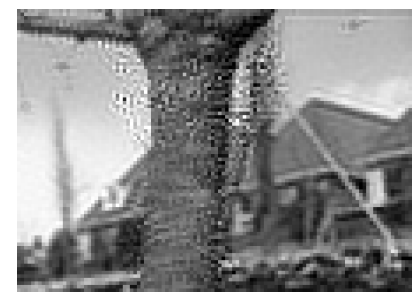

(c) SR-BOR $(22.9 \mathrm{~dB})$

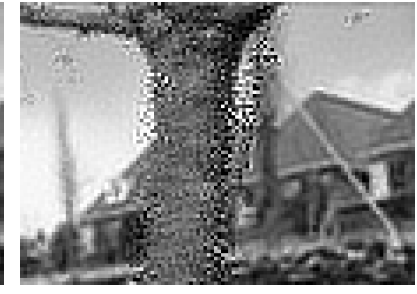

(b) SR-TRA (18.7 dB)

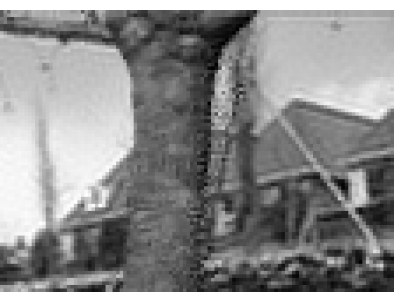

(d) SR-SIM (25.5 dB)
Figura 12. Resultados visuais da imagem 3 da sequiência Flower Garden (movimento estimado), com norma $\ell_{2}$, detecção de outliers desativada e modelos de primeira ordem.

Entretanto, os valores na Tabela 4, indicam que o algoritmo proposto, com norma Huber, é mais robusto aos outliers. Observe que a perda de qualidade com a detecção desativada foi mínima no método proposto quando comparada com a perda de qualidade dos algoritmos SR-BOR e SR-TRA. Esta perda de qualidade significativa nos métodos SR-BOR e SR-TRA é ocasionada pela presença dos outliers do modelo combinado no termo de dados, onde a norma $\ell_{2}$ ao quadrado está sendo utilizada. No algoritmo proposto neste trabalho o termo de dados não contém o modelo combinado, logo, não é influenciado pelos outliers. Os resultados visuais das figuras 13 e 14 mostram que as distorções na região dos outliers não são significativas nos resultados do SR-SIM, contudo afetam 
significativamente os resultados do SR-BOR e SR-TRA, confirmando a robustez do método simultâneo com norma Huber proposto neste trabalho, conforme comentado em detalhes na seção 5.3.

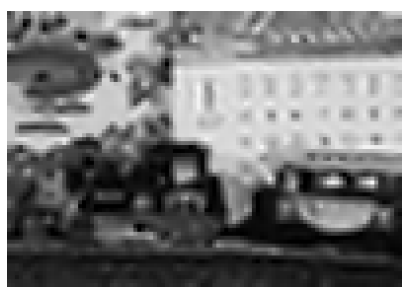

(a) SR-UIM (20.9 dB)

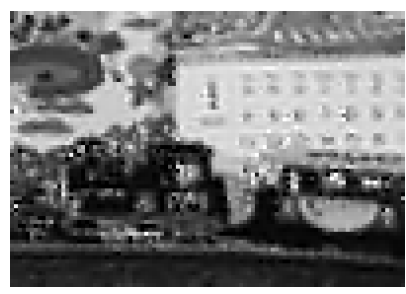

(c) SR-BOR (20.5 dB)

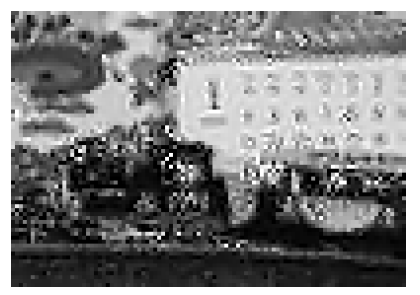

(b) SR-TRA (16.9 dB)

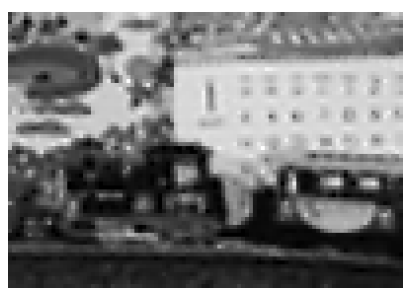

(d) SR-SIM $(22.0 \mathrm{~dB})$
Figura 13. Resultados visuais da imagem 3 da seqüência Mobile Calendar (movimento estimado), com norma Huber, detecção de outliers desativada e modelos de segunda ordem.

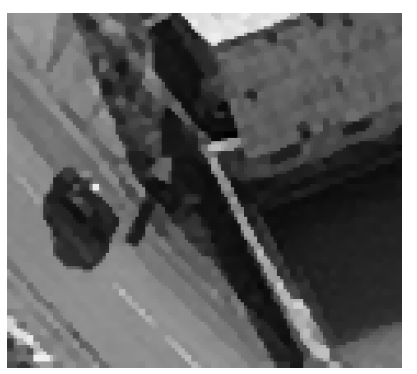

(a) SR-UIM (30.4 dB)

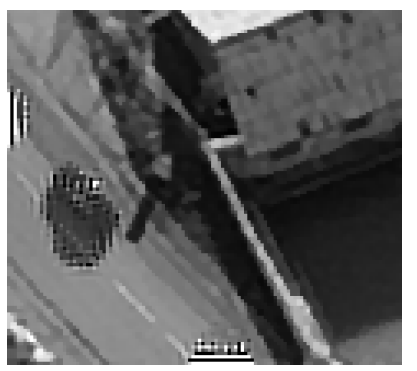

(c) SR-BOR (14.7 dB)

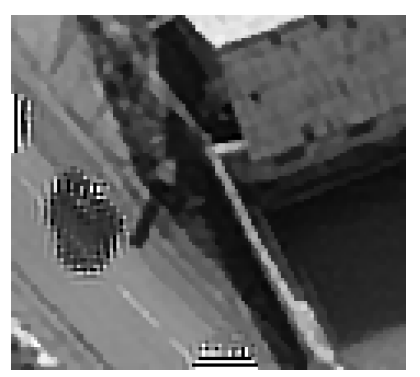

(b) SR-TRA (12.2 dB)

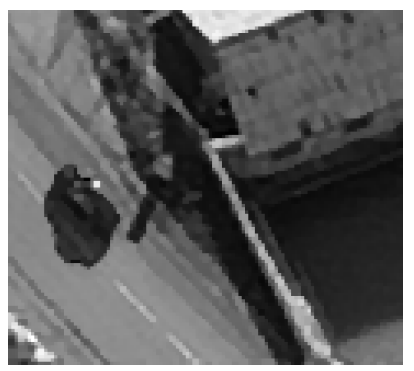

(d) SR-SIM (33.3 dB)
Figura 14. Resultados visuais da imagem 2 da sequiência Estrada (movimento conhecido), com norma Huber, detecção de outliers desativada e modelos de primeira ordem.

Através da observação dos valores na Tabela 5, conclui-se que o algoritmo com menor custo é o algoritmo de única imagem. Entretanto, este algoritmo apresenta resultados com menor fidelidade. Os algoritmos tradicionais e o algoritmo proposto apresentam custo computacional similar, porém o método proposto apresentou uma qualidade superior. Podese observar que o custo computacional dos algoritmos que re- alizam a minimização através do método de Gradiente Conjugado é bem menor do que o custo dos algoritmos que utilizam a minimização através do método de Gradiente Descente. Os resultados na Tabela 5 mostram que o custo do algoritmo com norma Huber ainda é significativamente maior do que o custo dos algoritmos com norma $\ell_{2}$.

Conclui-se, assim, que o método proposto oferece resultados de alta qualidade, similar ao algoritmo simultâneo SRBOR, porém com custo computacional menor, equivalente ao custo dos métodos tradicionais. Além disso, o algoritmo proposto, com penalidade Huber, possui uma robustez aos outliers muito maior do que a dos demais algoritmos testados.

\section{CONCLUSÕES}

Neste trabalho é proposta uma nova classe de algoritmos de super-resolução que recupera as imagens da sequiência de forma simultânea. Os algoritmos propostos são baseados nos métodos regularizados de super-resolução. Esta nova classe de algoritmos se distingue das demais pela utilização do movimento somente no termo a priori. O método proposto é comparado com outros métodos clássicos existentes na literatura e os resultados dos experimentos indicam claramente que o método proposto produz imagens com alta qualidade e com menor custo computacional. Além disso, o método proposto, com norma Huber, apresentou uma maior robustez aos outliers existentes no movimento.

\section{REFERÊNCIAS}

[1] Subhasis Chaudhuri. Super-Resolution Imaging. The Kluwer International Series in Engineering and Computer Science. Kluwer, 2001.

[2] Sung Cheol Park, Min Kyu Park, and Moon Gi Kang. Superresolution image reconstruction: a technical overview. IEEE Signal Processing Magazine, 20(3):21-36, May 2003.

[3] M. Bertero and P. Boccacci. Super-resolution in computational imaging. Micron, 34(6):265-273, October 2003.

[4] Sean Borman and Robert L. Stevenson. Spatial resolution enhancement of low-resolution image sequences. a comprehensive review with directions for future research. Technical report, University of Notre Dame, 1998.

[5] Sean Borman and Robert L. Stevenson. Super-resolution from image sequences - a review. In Proc. 1998 Midwest Symp. Circuits and Systems, pages 374-378, 1999.

[6] Marcelo V. W. Zibetti and Joceli Mayer. Adaptive regularization for multiframes resolution restoration considering subpixel contributions. In Anais Simpósio Brasileiro de Telecomunicações, Rio de Janeiro, Brazil, October 2003.

[7] Marcelo V. W. Zibetti. Restauração da resolução de seqüência de imagens usando regularização adaptativa. Master's thesis, Universidade Federal de Santa Catarina, February 2003.

[8] Michael Elad and Aire Feuer. Super-resolution reconstruction of image sequences. IEEE Transactions on Pattern Analysis and Machine Intelligence, 21(9):817-834, September 1999.

[9] Javier Mateos, Aggelos K. Katsaggelos, and Rafael Molina. Resolution enhancement of compressed low reolution video. In IEEE International Conference on Acoustics, Speech, and Signal Processing, volume 4, pages 1919-1922, June 2000.

[10] Sean Borman and Robert L. Stevenson. Simultaneous multiframe map super-resolution video enhancement using spatio- 
temporal priors. In IEEE International Conference on Image Processing, volume 3, pages 469-473, October 1999.

[11] Marcelo V. W. Zibetti and Joceli Mayer. Simultaneous superresolution for video sequences. In IEEE International Conference on Image Processing, volume 1, pages 877-880, September 2005.

[12] Harrison H. Barrett and Kyle J. Myers. Foundations of Image Science. Wiley Series in Pure and Applied Optics. John Wiley \& Sons, 2004.

[13] Yousef Saad. Iterative methods for sparse linear systems. International Thompson Publishing, 1995.

[14] Christoph Stiller and Janusz Konrad. Estimating motion in image sequences. IEEE Signal Processing Magazine, 16(4):7091, July 1999.

[15] Richard R. Schultz, Li Meng, and Robert L. Stevenson. Subpixel motion estimation for super-resolution image sequence enhancement. Journal of Visual Communication and Image Representation, 9(1):38-50, March 1998.

[16] P. J. Huber. Robust Statistics. John Wiley \& Sons, 1981.

[17] David Hasler, Luciano Sbaiz, Sabine Süsstrunk, and Martin Vetterli. Outlier modeling in image matching. IEEE Transactions on Pattern Analysis and Machine Intelligence, 25(3):301-315, March 2003.

[18] Richard R. Schultz and Robert L. Stevenson. Extraction of high-resolution frames from video sequences. IEEE Transactions on Image Processing, 5(6):996-1011, June 1996.

[19] Curtis R. Vogel. Computational Methods for Inverse Problems. Frontiers in Applied Mathematics. SIAM, 2002.

[20] Per Christian Hansen, editor. Rank-Deficient and Discrete IllPosed Problems. SIAM Monoghraphs an Matematical Modeling and Computation. SIAM, 1998.

[21] Gerhard Winkler. Image analysis, random fields and Markov chain Monte Carlo methods: a mathematical introduction. Applications of mathematics. Springer-Verlag, 2nd edition edition, 2003.

[22] Ali Mohammad-Djafari. From deterministic to probabilistic approaches to solve inverse problems. In Bayesian Inference for Inverse Problems, SPIE 98, pages 2-11, 1998.

[23] Al Bovik, editor. Handbook of Image and Video Processing. Academic Press, 2000.

[24] Anuj Srivastava. Stochastic models for capturing image variability. IEEE Signal Processing Magazine, 19(5):63-76, September 2002.

[25] Robert L. Stevenson, Barbara E. Schmitz, and Edward J. Delp. Discontinuity preserving regularization of inverse visual problems. IEEE Transactions on Systems, Man and Cybernetics, 24(3):455-469, March 1994.

[26] Charles Bouman and Ken Sauer. A generalized gaussian image model for edge-preserving map estimation. IEEE Transactions on Image Processing, 2(3):269-310, July 1993.

[27] Phelippe Thévenaz, Thierry Blu, and Michael Unser. Handbook of Medical Imaging, Processing and Analysis, chapter Image Interpolation and Resampling, pages 393-420. Academic Press, San Diego Ca, USA, 2000.

[28] Phelippe Thévenaz, Thierry Blu, and Michael Unser. Interpolation revisited. IEEE Transactions on Medical Imaging, 19(7):739-758, July 2000.

[29] Richard R. Schultz and Robert L. Stevenson. A bayesian approach to image expansion for improved definition. IEEE Transactions on Image Processing, 3(3):233-242, May 1994.

[30] S.E. El-Khamy, M.M. Hadhoud, M.I. Dessouky, B.M. Salam, and F.E. Abd El-Samier. Efficient implementation of image interpolation as an inverse problem. Digital Signal Processing, 15(2):137-152, March 2005.

[31] Mokhatar S. Bazaraa, Hanif D. Sherali, and C. M. Shetty. Non- linear Programming: Theory and Algorithms. John Wiley \& Sons, 2 edition, 1993.

[32] Jonathan Richard Shewchuk. An introdution to conjugate gradient method without the agoning pain. Technical report, Carnegie Mellon University, 1994.

[33] P. Erhan Eren, M. Ibrahim Sezan, and A Murat Tekalp. Robust, object-based high-resolution image reconstruction from low-resolution video. IEEE Transactions on Image Processing, 6(10):1446-1451, October 1997.

[34] Berthold K. P. Horn and Brian G. Schunck. Determining optical flow. Artificial Intelligence, 17:185-203, August 1981.

Marcelo Victor Wüst Zibetti recebeu o grau de Bacharel em Eng. Elétrica, em 2001, da Universidade de Passo Fundo e o grau de Mestre em Eng. Elétrica em 2003 da Univeridade Federal de Santa Catarina, Florianópolis. Atualmente, ele está concluindo o Doutorado em Eng. Elétrica pela Univeridade Federal de Santa Catarina, na área de Comunicações e Processamento de Sinais.

Joceli Mayer recebeu o grau de Bacharel em Eng. Elétrica, em 1988, e o grau de Mestre em Eng. Elétrica, em 1991, da Univeridade Federal de Santa Catarina. Ele também recebeu o grau de Mestre em Ciências, em 1998, e Ph.D., em 1999, em Engenharia da Computação da Universidade da Califórnia em Santa Cruz (E.U.A). Ele é Professor do Departamento de Engenharia Elétrica, na Universidade Federal de Santa Catarina, desde 1993. Professor Joceli Mayer atua nas áreas de Processamento Digital de Sinais, Voz, Imagens e Vídeo. Homepage: http://eel.ufsc.br/ mayer. 